\title{
AdS/CFT correspondence and coincident D-6-branes
}

\author{
Sergei V. Ketov \\ Institut für Theoretische Physik, Universität Hannover \\ Appelstraße 2, 30167 Hannover, Germany
}

\begin{abstract}
A relation between confinement and Maldacena conjecture is briefly discussed. The gauge symmetry enhancement for two coincident D-6-branes is analyzed from the viewpoint of the hypermultiplet low-energy effective action given by the $\mathrm{N}=2$ supersymmetric non-linear sigma-model with the Eguchi-Hanson (ALE) target space.
\end{abstract}

The most attractive mechanism of color confinement in QCD is known to be the dual (type II) superconductivity, i.e. a creation of color-electric fluxes (or strings) having quarks at their ends [1, 2]. The usual type-II superconductivity (i.e. the confinement of magnetic charges) is known to be a solution to the standard Landau-Ginzburg theory, whereas the QCD confinement is supposed to be a non-perturbative solution to a $(1+3)$ dimensional quantum $S U\left(N_{c}\right)$ gauge field theory with $N_{c}=3$.

The formal proof of the confinement in QCD amounts to a derivation of the area law for a Wilson loop $W[C]$. It may be based on the following 'string' Ansatz [3]:

$$
W[C] \sim \int_{\substack{\text { surfaces } B \\ \partial B=C}} \exp \left(-S_{\text {string }}\right) \quad .
$$

Eq. (1) clearly shows that the effective degrees of freedom (or collective coordinates) in QCD at strong coupling (in the infra-red) are strings whose worldsheets are given by surfaces $B$ and whose dynamics is governed by (still unknown) action $S_{\text {string. The }}$ fundamental (Schwinger-Dyson) equations of QCD can be reformulated into the equivalent infinite chain of equations for the Wilson loops [4]. The chain of loop equations drastically simplifies at large number of colors $N_{c}$ (i.e. when only planar Feynman graphs are taken into account) to a single closed equation known as the Makeenko-Migdal loop equation [5]. Eq. (1) is, therefore, just the Ansatz for a solution to the MM loop equation in terms of some string action $S_{\text {string }}$ to be determined out of it. The main (yet unsolved) problems in a realization of this program in the past were (i) taking into account quantum renormalization in the MM equation, and (ii) determining the corresponding string action [6]. The first problem does not arise if one replaces QCD by the $\mathrm{N}=4$ supersymmetric Yang-Mills (SYM) theory and considers the $\mathrm{N}=4$ supersymmetric MM-type loop equation instead of the original $(\mathrm{N}=0)$ one, just because of the well-known fact that, being a scale invariant quantum field theory in $1+3$ spacetime dimensions, the $\mathrm{N}=4 \mathrm{SYM}$ does not renormalize at all. The recent Maldacena conjecture [7], claiming that the N=4 SYM theory is dual to the IIB superstring theory in the $A d S_{5} \times S^{5}$ background, can then be interpreted as the particular Ansatz $S_{\text {string }}=S_{I I B / A d S_{5} \times S^{5}}$ for a solution to the N=4 
super-MM loop equation in the form of eq. (1). Within the Maldacena conjecture, the $(1+3)$-dimensional spacetime is identified with the boundary of the anti-de-Sitter space $A d S_{5}$, where

$$
A d S_{5}=\frac{S O(4,2)}{S O(4,1)} \quad \text { and } \quad S^{5}=\frac{S O(6)}{S O(5)}
$$

while the coupling constants are related to the $A d S_{5}$ radius as follows [7]:

$$
\left(\alpha^{\prime}\right)^{-2} R_{\mathrm{AdS}}^{4} \sim g_{\mathrm{YM}}^{2} N_{c}, \quad g_{\text {string }} \sim g_{\mathrm{YM}}^{2} .
$$

The proposed duality is a strong-weak coupling duality:

- for small $\lambda=g_{\mathrm{YM}}^{2} N_{c}$ a perturbative SYM description applies,

- for large $\lambda$ a perturbative IIB string/AdS supergravity description applies.

It is in agreement with the holographic proposal [8] since physics in the $A d S_{5}$ bulk is supposed to be encoded in terms of the field theory defined on the $A d S_{5}$ boundary. The quantum $\mathrm{N}=4 \mathrm{SYM}$ theory is conformally invariant, while its rigid symmetry is given by the supergroup $S U(2,2 \mid 4)$ that contains 32 supercharges. The isometries of $A d S_{5} \times S^{5}$ form the group $S O(4,2) \times S O(6) \cong S U(2,2) \times S U(4)$ whose extension in the AdS supergravity is also given by $S U(2,2 \mid 4)$. In addition, both the $\mathrm{N}=4 \mathrm{SYM}$ and type-IIB superstrings are believed to be self-dual under the S-duality group $S L(2, \mathbf{Z})$. In more practical terms, this CFT/AdS correspondence is just a one-to-one correspondence [9] between the N=4 SYM correlators and the correlators of the certain string theory whose action $S_{\text {string }}$ is known and whose correlators can be computed, in principle, by the methods of two-dimensional conformal field theory [10]. Quantum corrections in powers of $\left(\alpha^{\prime} \times\right.$ curvature $)$ on the string theory side correspond to corrections in powers of $\left(g_{\mathrm{YM}}^{2} N_{c}\right)^{-1 / 2}$ on the gauge field theory side, while the string loop corrections are suppressed by powers of $N_{c}^{-2}$.

The close connection between IIB strings and $\mathrm{N}=4 \mathrm{SYM}$ is also known to exist within the modern brane technology. The type-IIB supergravity (= the low-energy effective field theory of IIB strings) admits extended solitonic BPS-like classical solutions known as D-3-branes and D-strings (or 'mesons') [11]. These solutions can spontaneously break the conformal invariance in the non-perturbative $\mathrm{N}=4 \mathrm{SYM}$ theory, and thus may be useful for a simulation of confinement. When $N_{c}$ parallel and similarly oriented D-3branes coincide, the low-energy effective field theory action in their common worldvolume appears to be the $\mathrm{N}=4 \mathrm{SYM}$ with the gauge group $U\left(N_{c}\right)$ [12. The brane picture thus provides a classical resolution to the non-perturbative $\mathrm{N}=4 \mathrm{SYM}$, in the very similar way as the M-theory 5-brane classical dynamics yields exact Seiberg-Witten-type solutions to $\mathrm{N}=2$ supersymmetric quantum gauge field theories (see e.g., ref. [13 for a review). The 11-dimensional M-theory compactified on a 2-dimensional torus is known to be dual to the 10-dimensional type-IIB strings compactified on a circle [14], so that the relevant phenomenon is just the gauge symmetry enhancement for coincident D-branes alone. We would like to understand it from the field-theoretical point of view, and distinguish between those elements of this phenomenon that are of perturbative origin and those elements that are truly non-perturbative.

As an example, consider the case of two nearly coincident KK monopoles in M theory. The metric is essentially given by the 2-centre Taub-NUT metric (or, equivalently, the mixed Taub-NUT-Eguchi-Hanson metric) characterized by the harmonic potential [15]

$$
H(\vec{y})=\lambda+\frac{1}{2}\left\{\frac{1}{|\vec{y}-\xi \vec{e}|}+\frac{1}{|\vec{y}+\xi \vec{e}|}\right\} .
$$


In the limit $\xi \rightarrow 0$ the homology 2-sphere connecting two KK monopoles contracts to a point. Since the energy of an M-2-brane wrapped about this 2-sphere is proportional to its area (of order $\xi$ ), a massless vector particle (= the zero-mode of the M-2-brane) appears. In the type-IIA picture, the ground states of the 6-6 strings connecting two D6-branes become massless if the branes coincide. The net effect is called the non-abelian gauge symmetry enhancement: $U(1) \times U(1) \rightarrow U(2)$, and it is truly non-perturbative [16]. The gauge fields (and their supersymmetric partners) related to non-diagonal gauge symmetry generators are the ground states of strings connecting different D-6-branes, with the masses being proportional to the distance between the D-branes, whereas those related to the Cartan subalgebra generators appear as the massless ground states of the strings ending on the same D-6-brane. The existence of the massless ground states associated with Cartan subalgebra can be understood perturbatively, as a result of dynamical generation of massless vector supermultiplets in the (one-loop) quantum perturbation theory [17].

The effective field theory in the D-6-brane worldvolume dimensionally reduced to four dimensions includes the hyper-Kähler non-linear sigma-model (NLSM) for a selfinteracting hypermultiplet, whose NLSM metric is dictated by the potential (4). This NLSM can be written down in harmonic superspace, in terms of two hypermultiplets $q^{A+}$, $A=1,2$, and the auxiliary $\mathrm{N}=2$ vector superfield $V^{++}$as a Lagrange multiplier,

$$
S_{\text {mixed }}\left[q^{A}, V^{++}\right]=\int_{\text {analytic }}\left\{\bar{q}^{A+} D_{Z}^{++} q_{A}^{+}+V^{++}\left(\frac{1}{2} \varepsilon^{A B} \bar{q}_{A}^{+} q_{B}^{+}+\xi^{++}\right)+\frac{1}{4} \lambda\left(\bar{q}^{A+} q_{A}^{+}\right)^{2}\right\},
$$

where the Fayet-Iliopoulos term $(\sim \xi V)$ has been introduced, while both hypermultiplets are supposed to have different masses, $m_{1}$ and $m_{2}$, given by the $\mathrm{N}=2$ central charge $(Z)$ eigenvalues. Eq. (5) is the gauged NLSM over the non-compact coset space $S U(1,1) / U(1)$ parametrized by two hypermultiplets. Near the core of D-6-branes the parameter $\lambda$ becomes irrelevant, so that the NLSM target space looks like an ALE space with the Eguchi-Hanson metric. Setting $\lambda=0$ in eq. (5) results in a formally renormalizable fourdimensional 'linear' NLSM, that allows us to integrate over the hypermultiplets in eq. (5). All one needs is the $\mathrm{N}=2$ superfield hypermultiplet propagator in harmonic superspace [18],

$$
i\left\langle q^{+}(1) \bar{q}^{+}(2)\right\rangle=-\frac{1}{\square_{1}^{Z}}\left(D_{1}^{+}\right)^{4}\left(D_{2}^{+}\right)^{4}\left\{\delta^{12}\left(Z_{1}-Z_{2}\right) \frac{e^{v_{Z}(2)-v_{Z}(1)}}{\left(u_{1}^{+} u_{2}^{+}\right)^{3}}\right\},
$$

where $\square^{Z}$ is the Klein-Gordon operator, and $v_{Z}$ is the co-called 'bridge' (see ref. [18] for details). It is now straightforward to calculate the one-loop gauge effective action $i \operatorname{Tr} \log \left(\mathcal{D}_{Z, V}^{++}\right)$in the low-energy approximation [17]. We find that the $\mathrm{N}=2$ vector supermultiplet, introduced in the classical action (5) as the Lagrange multiplier (without a kinetic term), becomes dynamical in quantum theory due to a dynamical generation of its kinetic term

$$
S_{\text {induced }}\left[V^{++}\right]=-\frac{1}{2 e_{\text {ind }}^{2}(p)} \int_{\text {chiral }} W^{2}+\text { h.c. },
$$

where we have introduced the standard $\mathrm{N}=2$ superfield strength $W=\int d u\left(\bar{D}^{-}\right)^{2} V^{++}$. The induced (momentum-dependent) gauge coupling constant appears to be [17]

$$
\frac{1}{e_{\mathrm{ind}}^{2}(p)}=\frac{1}{16 \pi^{2}} \int_{0}^{1} d x \ln \frac{m_{2}^{2}+p^{2} x(1-x)}{m_{1}^{2}+p^{2} x(1-x)}=\frac{1}{e_{0}^{2}}+O\left(p^{2} / m^{2}\right) .
$$

The $\mathrm{N}=2$ central charge providing masses to the hypermultiplets can, therefore, be understood as the origin of the induced gauge coupling (8). 
A generalization to higher unitary groups is straightforward [17]. The Hooft limit of large $\lambda$ appears to be equivalent to $\left|\xi / Z^{2}\right| \rightarrow 0$ in our approach. The orthogonal gauge groups may also be considered by introducing orientifolds and the Atiyah-Hitchin metric in M-theory.

\section{References}

[1] G. t'Hooft, contributed to the Proceedings of the EPS Intern. Conference in Palermo, Italy, 23-28 June, 1975; in 'High Energy Physics', Vol. 1, ed. by A. Zichichi, Bologna, 1976

[2] S. Mandelstam, Phys. Rep. 23 (1976) 245

[3] A. M. Polyakov, Gauge Fields and Strings, Harwood Academic Publishers, Chur, Switzerland, 1987; The wall of the cave, hep-th/9809057

[4] A. Migdal, Phys. Rep. 102 (1983) 199

[5] Y. Makeenko and A. Migdal, Nucl. Phys. B188 (1981) 269

[6] A. Migdal, private communication

[7] J. Maldacena, The large $N$ limit of superconformal field theories and supergravity, hep-th/9711200

[8] L. Susskind, The world as a hologram, hep-th/9409089

[9] S. S. Gubser, I. R. Klebanov and A. M. Polyakov, Phys. Lett. 428B (1998) 105

[10] S. V. Ketov, Conformal Field Theory, Singapore, World Scientific, 1996

[11] G. T. Horowitz and A. Strominger, Nucl. Phys. B360 (1991) 197

[12] E. Witten, Nucl. Phys. B460 (1996) 335

[13] S. V. Ketov, Analytic tools to brane technology, hep-th/9806009

[14] J. H. Schwarz, Phys. Lett. 360B (1995) 13, ibid. 364 (1995) 252

[15] P. K. Townsend, Phys. Lett. 350B (1995) 184

[16] H. Ooguri and C. Vafa, Nucl. Phys. B463 (1996) 55

[17] S. V. Ketov, Dynamical generation of vector and Higgs bosons in N=2 supersymmetric non-linear sigma-models, hep-th/9808187

[18] E. A. Ivanov, S. V. Ketov and B. M. Zupnik, Nucl. Phys. B509 (19998) 53. 